\title{
Criminologie
}

\section{La démocratie et les paradoxes du discours sur la torture au Brésil}

\section{Mariana Thorstensen Possas}

Volume 45, numéro 1, printemps 2012

Violence politique et conflits armés

URI : https://id.erudit.org/iderudit/1008380ar

DOI : https://doi.org/10.7202/1008380ar

Aller au sommaire du numéro

\section{Éditeur(s)}

Les Presses de l’Université de Montréal

ISSN

0316-0041 (imprimé)

1492-1367 (numérique)

Découvrir la revue

Citer cet article

Possas, M. T. (2012). La démocratie et les paradoxes du discours sur la torture au Brésil. Criminologie, 45(1), 137-151. https://doi.org/10.7202/1008380ar
Résumé de l'article

En dépit du fait que la pratique de la torture dans les sociétés contemporaines occidentales est à première vue une pratique inacceptable, lorsqu'on observe les discours de plus près, on constate qu'il existe en fait deux discours : l'un qui légitime la torture et l'autre qui la refuse. Le présent article porte sur le discours qui rejette la pratique de la torture et sur la « construction du sens du problème " (Bachelard, 1938) de la torture au Brésil et ses explications. Ces dernières tournent autour de la croyance selon laquelle la persistance de la pratique de la torture au Brésil, après l'avènement de la démocratie dans les années 1980, est due à la faible consolidation démocratique. Ce type de raisonnement contient des taches aveugles ("blind spots »), qui nous empêchent de voir des aspects importants, comme le fait que la pratique de la torture n'est pas une réalité exclusive des régimes autoritaires mais aussi, malheureusement, des démocraties.
Ce document est protégé par la loi sur le droit d'auteur. L'utilisation des services d’Érudit (y compris la reproduction) est assujettie à sa politique d'utilisation que vous pouvez consulter en ligne.

https://apropos.erudit.org/fr/usagers/politique-dutilisation/ 


\title{
La démocratie et les paradoxes du discours sur la torture au Brésil
}

\author{
Mariana Thorstensen Possas \\ Université de São Paulo, Brésil \\ marianapossas@gmail.com
}

\begin{abstract}
RÉSUMÉ - En dépit du fait que la pratique de la torture dans les sociétés contemporaines occidentales est à première vue une pratique inacceptable, lorsqu'on observe les discours de plus près, on constate qu'il existe en fait deux discours: l'un qui légitime la torture et l'autre qui la refuse. Le présent article porte sur le discours qui rejette la pratique de la torture et sur la "construction du sens du problème» (Bachelard, 1938) de la torture au Brésil et ses explications. Ces dernières tournent autour de la croyance selon laquelle la persistance de la pratique de la torture au Brésil, après l'avènement de la démocratie dans les années 1980, est due à la faible consolidation démocratique. Ce type de raisonnement contient des taches aveugles ("blind spots»), qui nous empêchent de voir des aspects importants, comme le fait que la pratique de la torture n'est pas une réalité exclusive des régimes autoritaires mais aussi, malheureusement, des démocraties.
\end{abstract}

MOTS-CLÉS - Torture, consolidation démocratique, violence policière, Brésil.

\section{Introduction}

Le présent article est une réflexion sur la construction des discours sur la torture qui sont tenus au Brésil avec pour toile de fond la démocratie politique. Plusieurs ont cru (ou espéré) que la fin du régime autoritaire caractérisé par des actions violentes et arbitraires et la transition à la démocratie dans les années 1980 se traduiraient par la disparition de la torture. Or on commence à constater que ce n'est pas le cas, que la torture continue bel et bien d'exister dans les postes de police et dans les prisons et qu'elle est pratiquée surtout par les policiers ou par d'autres agents de l'État. L'objectif de cet article est de faire une analyse des communications qui portent sur ce problème et d'en dégager les paradoxes afférents. 
Une grande partie du contenu de ce travail est extraite de notre recherche doctorale, laquelle portait sur le processus politique de création de la loi contre la torture au Brésil. Entre autres choses, nous avons observé un phénomène, déjà soulevé par Pires dans d'autres contextes (Pires et Garcia, 2007; Pires, 2008), concernant la mobilisation des droits de la personne: devant certaines situations considérées comme de graves violations des droits de la personne, telle la torture, la mobilisation de cette trame discursive (des droits de la personne) a toujours été associée à la demande de peines sévères de prison, sans aucun questionnement ou recul critique par rapport à ce type de réponse. En d'autres mots, on peut dire que la mobilisation des droits de la personne est, dans le cas étudié dans ma recherche, associée à la mobilisation de la rationalité pénale moderne (Pires, 1998).

En ce qui a trait au matériel empirique, nous avons repris pour ce travail deux types de données recueillies lors de la réalisation du terrain de ma recherche doctorale: des articles de journaux portant sur la question de la création d'une loi spécifique sur la torture et des extraits d'entretiens réalisés auprès de politiciens qui ont participé de façon directe ou indirecte à la passation de la loi contre la torture au Parlement, ainsi qu'auprès de membres de mouvements sociaux préoccupés par cette question au Brésil.

\section{Deux trames discursives sur la torture dans la société contemporaine}

Pour quelques secteurs des sciences sociales, du droit ou du point de vue de la morale, la torture est, à première vue, un thème sans controverse. Sur le plan normatif, son interdiction revêt un caractère de norme absolue, qui n'accepte pas d'exceptions ou de relativisations. Spécialement sur le plan du droit international, l'interdiction de la torture est vue comme un acquis majeur (Babissagana, 2006).

Or, le fait est que la torture a toujours existé et continue à être infligée dans les pays qui se disent les plus démocratiques et les défenseurs les plus combatifs des droits de la personne (Lehalle, 2008). Et non seulement la torture existe-t-elle, mais elle est pratiquée de façon continue, organisée et (même si non assumée) comme élément des politiques gouvernementales de sécurité publique (j’y reviendrai) dans le cadre de la criminalité «ordinaire» (par opposition à des attentats terroristes, ou même à la criminalité des cols blancs). Et ce fait n'empêche 
pas les pays qui y recourent d'avoir un siège à la Commission des droits de l'Homme des Nations Unies (Jacques, 1994: 84). Bref, loin d'être en voie de disparition, la torture est plutôt en voie de globalisation. En effet, on semble assister à la renaissance d'une véritable «culture de la torture», sous prétexte de la «lutte contre le terrorisme» (Babissagana, 2006: 12) ${ }^{1}$.

La réalité de la persistance de la torture comme instrument du pouvoir m'amène à interroger l'idée de l'existence d'un seul discours homogène ou d'une seule position s'affichant contre la torture dans les pays occidentaux, ainsi qu'au Brésil. Et effectivement, mes données empiriques confirment l'hypothèse de l'acceptation «discursive» de la torture (qui ne se limite pas à la pratique), aussi surprenant que cela puisse paraitre. Bref, il ne s'agit pas exclusivement d'un problème ou d'une opposition entre norme (le «devoir-être») et pratique («ce qui se passe dans le monde réel»), mais entre deux types de discours différents.

Dans le cadre de la lutte contre le terrorisme, Lehalle (2008) a recensé quelques exemples d'argumentations juridiques proposées par les avocats-conseils au président des États-Unis. Ces documents avaient pour objectif d'encadrer légalement les pratiques qui détournent l'interdit de la torture. On lit ainsi des justifications pour la non-application $\mathrm{du}$ droit d'babeas corpus dans certains cas, pour la non-application des traités internationaux lorsqu'il s'agit de détenus d'Al-Qaeda et de talibans ou même pour l'utilisation de certaines techniques d'interrogation (telles cagoules, nudité, phobies des prisonniers, menaces de mort, exposition au froid et à l'eau, etc.) considérées, par ces documents, comme n'étant pas des pratiques de «torture».

$\mathrm{Du}$ point de vue des pratiques, la torture est perçue par plusieurs personnes au Brésil comme une question actuelle et sérieuse, toujours présente dans plusieurs pays en dépit de l'adoption de traités internationaux. Néanmoins, la pratique de la torture ici est, différemment des États-Unis, actuellement apparentée surtout à la brutalité policière contre les criminels «communs» (non terroristes). Les taux croissants de la criminalité sont en grande partie perçus comme un problème qui

1. Aujourd'hui, il y a une énorme littérature sur le sujet de la torture, notamment aux États-Unis, depuis les attentats du 11 septembre et l'accroissement de la politique antiterroriste menée par le président George W. Bush. Je ne vais néanmoins pas aborder cette littérature étant donné qu'elle traite, en grande partie, du cas étatsunien et de ses particularités. 
doit être combattu à n'importe quel prix, ce qui légitime, à la limite, toutes sortes d'actions destinées à diminuer le crime ${ }^{2}$. La demande de punition des actes arbitraires perpétrés par la police est ainsi diluée et, en même temps, le mouvement pour la défense des droits de la personne, en ce qui concerne les criminels, est fréquemment vu comme un mouvement pour la "protection des bandits». Un juge, au Brésil, en acquittant des policiers accusés de torture a écrit: «Ces droits de la personne existent seulement pour protéger les criminels de la loi, lorsqu'en vérité ils doivent protéger les citoyens honnêtes des actions des bandits» (Human Rights Watch, 1997: 2; notre traduction).

Les recherches d'opinion montrent, par ailleurs, que la brutalité policière, lorsqu'elle est exercée contre les «bandits», notamment ceux appartenant aux classes sociales moins aisées, est en effet largement acceptée. Costa (2004), par exemple, mentionne une recherche menée à la ville de Rio de Janeiro, dont les résultats indiquent qu'une partie significative de la population trouve que la solution à la violence urbaine réside dans l'action énergique de la police. Spécifiquement en ce qui concerne la torture, cette enquête, réalisée en 1997, a constaté qu'à peu près $44 \%$ de la population accepte la pratique de la torture dans les postes de police, en vue d'obtenir des aveux.

Dans le livre de semi-fiction Elite da tropa (L'élite de la troupe) dont l'objectif était de rendre publiques ainsi que de dénoncer la violence policière et la manière de travailler de la police à Rio de Janeiro, on trouve des passages sur des événements concernant la routine de quelques policiers appartenant audit bataillon et quelques réflexions rapides sur leurs pratiques, comme celle de la torture pratiquée contre les «bandits»:

\begin{tabular}{|l|l|l|}
\hline 1 & Le sujet est la violence. C'est-à-dire la violence que nous com- & \\
& mettons. Quelques-uns l'appellent torture. Moi, je n'aime pas ce & \\
& mot, parce qu'il sous-entend une connotation diabolique. Je & Les \\
& pense qu'il y a des cas et des cas, et qu'on ne peut pas qualifier & "différents» \\
& de «torture» toutes les tortures, dans l'acception plus commune & types de \\
& du concept. [...] Ce que je veux dire est que je ne suis pas & violence \\
embarrassé de ne pas m'embarrasser du fait d'avoir battu des & contre les \\
& bandits, je n'ai tué que des criminels. [...] Et pour moi, un & criminels \\
& bandit est un bandit, soit-il un garçon ou un homme adulte. & \\
& (Soares et al., 2006: 35-36) & \\
\hline
\end{tabular}

2. Depuis les années 1980, le Brésil présente des taux croissants de criminalité. Le taux d'homicide, par exemple, est monté de 11,7 par 100000 habitants en 1980 à 26,2 en 1999. La violence est devenue la principale cause de mortalité selon les données du ministère de la Santé (Costa, 2004: 120). 
Dans cet extrait du livre on trouve une prise de position face à la torture: qu'elle vienne d'un narrateur fictif ou d'un policier réel, elle trouve un appui considérable auprès de quelques segments de la population du Brésil. Ce sont les mêmes segments d'ailleurs qui ont applaudi à la fin des projections cinématographiques de Tropa de Elite (film basé sur le livre Elite da Tropa), ou au passage des voitures du BOPE, dans les rues de Rio de Janeiro, lorsqu'elles sortaient des favelas, après une de leurs «opérations».

Ces exemples illustrent le fait que, du point de vue sociologique, il y aurait deux discours structurés par rapport à la torture: un qui la refuse complètement et un autre qui accepte la torture dans certaines situations où elle est vue comme nécessaire et ce, sans causer de problème moral à celui qui reproduit un tel discours. Ces deux trames discursives coexistent et se confrontent, sans que les individus en question reconnaissent ou acceptent l'existence de l'autre. Rappelons que je ne parle pas ici de la reconnaissance d'un discours marginal favorable à la torture, mais de l'existence d'un discours structuré qui la soutient et qui est donc beaucoup plus large que l'ensemble des points de vue de certains policiers «déviants» sur le sujet.

Cela est un exemple d'une «communication différentielle» par rapport à la loi criminelle, concept élaboré par Sutherland (1949). Selon Sutherland, on trouve dans la société contemporaine un ensemble de communications et de définitions de situations qui sont favorables ou défavorables à certains crimes. Ces communications circulent dans la société indépendamment des individus (des systèmes psychiques). Ainsi, chaque système psychique va sélectionner quelques communications et s'y associer. Les communications sélectionnées peuvent être contre ou en faveur du crime. La transmission (et l'apprentissage) des communications différentielles favorables au crime dépend de plusieurs circonstances, que je ne vais pas énumérer ici. L'importance de la théorie de Sutherland pour ce travail est l'observation que l'individu est capable d'apprendre et de valider pour luimême les communications favorables au crime, ainsi que les raisons ou les «motifs» pour adopter de telles communications (Debuyst et al., 2008).

Le cas du crime de torture offre un exemple empirique et relativement clair de communications différentielles sur la législation pénale, où chacun va sélectionner (ou s'associer à) des communications sur la torture qui lui semblent plus adéquates en général ou en fonction d'une 
situation déterminée, de sorte qu'on finit par avoir une dissonance par rapport à la légitimation de la torture. Il s'agit d'une norme de comportement qui semble créer une sorte de sélection éparpillée des communications favorables et non favorables.

\section{La perception du problème et les formulations des explications}

Sur le plan du discours qui la délégitime, la torture est vue comme un problème qui doit être combattu. On trouve cette perception dans plusieurs «circuits de communication» de la société brésilienne, y compris les médias, la doctrine juridique et les mouvements pour la défense des droits de la personne. Or, on y trouve des représentations variées de ce même problème et de ses causes. Je vais présenter quelques-unes de ces représentations sans prétendre en endosser aucune.

Par exemple, pour plusieurs observateurs, notamment les militants des droits de la personne, le diagnostic formulé pour expliquer la continuité des pratiques violentes des policiers passe très souvent par le problème de l'impunité dont ils jouissent, un problème dont le fonctionnement précaire du judiciaire serait le principal facteur (Chevigny, 1999: 57; Pinheiro (1999: 2-3). Ce type de diagnostic, très fréquent dans la littérature (sociologique) qui aborde le problème de la violence de la police et de la torture, est hautement sélectif par rapport aux causes. De plus, ces analyses attribuent normalement un poids énorme à la punition criminelle de ces actes, comme si le droit (le droit pénal en particulier) avait une capacité presque absolue de rendre compte d'une telle situation.

Parmi les explications sur l'existence de la torture dans le Brésil contemporain, on trouve un type de lecture des faits qui a tendance à attribuer l'origine de la torture actuelle, celle pratiquée par la police contre les criminels communs, à la période de la dictature militaire pendant laquelle la torture, pour des raisons politiques, a occupé le centre de l'attention. On lit, surtout dans les médias ou dans les manifestations de militants des droits de la personne, un positionnement critique quant à l'actuel État démocratique et à son incapacité de rendre compte du problème, comme si l'avènement du régime démocratique aurait dû signifier la fin de ce type de violation des droits de la personne. 


\section{La violence policière et la torture de criminels «ordinaires»}

S'il est vrai que la violence politique a beaucoup diminué avec l'avènement de la démocratie, un autre type de violence, non politique, est resté présent après la dictature. Les abus contre les droits de la personne ont continué à être perpétrés quotidiennement par les agents de l'État, mais désormais contre une autre cible: les criminels «communs» (par opposition à la criminalité en col blanc). Appartenant majoritairement aux couches les plus pauvres de la population, les criminels communs redeviennent les victimes les plus fréquentes des tortures et d'autres violences pratiquées par l'État.

À titre d'illustration de l'utilisation disproportionnelle, voire arbitraire de la force policière, on peut indiquer le haut nombre d'homicides perpétrés par la police militaire ${ }^{4}$. En 1992, ce chiffre a atteint une pointe de 1470 citoyens tués dans l'État de São Paulo, le plus riche du pays. Ce chiffre correspondait au tiers du total d'homicides qui ont eu lieu dans cet État et il était 61 fois plus élevé que le nombre de morts - 24 - par la police de New York au cours de la même année (Human Rights Watch, 1997: 13). Même avant 1992, le nombre de morts causées par la police militaire était déjà considéré comme très élevé.

Des données sur les conflits armés indiquent un rapport encore plus dramatique quand il s'agit de la violence envers des individus appartenant aux couches moins aisées. En 1990, dans la ville de São Paulo, la proportion était de 28 citoyens morts pour chaque policier tué lors de conflits armés avec la police. Si on se dirige vers les banlieues de São Paulo, connues pour leur pauvreté, la proportion monte à 40 pour 1 (40 citoyens morts pour chaque policier tué) (Costa, 2004).

Peu après octobre 1992, année où a eu lieu une tuerie dans la Maison de détention du Carandiru à São Paulo (111 prisonniers tués par la police après une émeute) - un fait que les médias ont répandu en faisant de la violence policière presque un symbole national et international -, les taux d'homicides commis par la police militaire ont commencé à tomber de manière substantielle. En 1996, le nombre de morts causées par la police (militaire) dans la ville de São Paulo était de 106, alors

3. Sur le sujet de la violence pratiquée par la police durant la période de la dictature militaire, voir Huggins et al. (2002).

4. L'institution de la police au Brésil est essentiellement divisée en deux grands groupes: la police civile, qui remplit les fonctions de police judiciaire, responsable de l'investigation criminelle, et la police militaire, responsable de la surveillance et de la préservation de l'ordre public. 
qu'il avait été de 1190 en 1992 (Human Rights Watch, 1997: 51). Même si cette tendance est vue comme positive, plusieurs sources indiquent que la violence policière persiste encore de façon préoccupante dans la ville et dans l'État de São Paulo et n'est pas du tout une question résolue.

\section{Démocratie et torture}

En dépit du fait que la torture existait bel et bien avant la dictature, j'observe une certaine tendance à oublier ou à ignorer cet aspect du problème. La violence policière qui a lieu dans le Brésil contemporain est vue par certains comme un des grands problèmes de la non-consolidation de la démocratie au Brésil, c'est-à-dire comme s'il s'agissait d'un problème qui surgit ou qui devient important à partir de la fin de la dictature. Cette lecture des faits indique une vision quelque peu «naïve» de la relation entre torture - démocratie - et dictature.

En effet, les actes de torture commis par des policiers constituent une réalité de la démocratie, notamment lors des activités d'investigation criminelle. Ces pratiques sont devenues tellement répandues qu'elles sont même considérées comme faisant partie de la vie ordinaire des institutions policières. Mendez (1999) soulève quelques raisons qui expliqueraient cet état de fait en Amérique latine. L'une d'elles est qu'il n'y aurait pas eu de changements significatifs au sein du personnel qui travaille dans les organes responsables de la sécurité, de sorte que plusieurs policiers qui, auparavant, s'occupaient de combattre la subversion, s'occupent aujourd'hui de la répression des crimes communs. Ainsi, l'héritage de l'utilisation de la violence contre les criminels ordinaires ferait encore partie de l'ethos de la police (Human Rights Watch, 1997: 21).

Ce type d'explication soulève un problème important: il donne l'impression, évidemment trompeuse, que le problème de la torture au Brésil serait une question reliée à la dictature. Encore une fois, c'est comme si avant la dictature (et avant le combat contre la subversion politique), la police n'avait pas torturé de manière significative et, après la dictature, elle avait arrêté de torturer en l'absence d'ennemis politiques. Le cœur du problème se trouverait alors dans la formation du policier qui, remontant à la dictature, l'inciterait à torturer les «communistes». Il s'agirait par conséquent d'une police inadaptée à la démocratie, qui doit être remplacée ou recevoir une nouvelle formation pour la répression des crimes «communs». 
Parmi les articles des médias traités datant de la période antérieure à la loi 9455/97 (loi contre la torture), la plupart vont se limiter à dénoncer des cas de torture, souvent en exprimant un certain étonnement face au constat que la torture, après la fin de la période autoritaire (durant les années 1980), "persiste» dans le quotidien des investigations policières ainsi que dans les prisons. Ces opinions, qui sont le fait autant des médias que des juristes ou des militants, ont comme dénominateur commun le fait de rendre évident à quel point la démocratie est incompatible avec la transgression de la dignité humaine que représente la torture. Les auteurs des articles en question assument que la (nouvelle) démocratie, en tant que nouveau régime politique qui se consolide au pays, ne peut pas accepter la torture, ils sous-entendent l'existence d'un lien entre la dictature et la torture, comme si celle-ci était une pratique propre à l'ancien régime.

Bien sûr, la période de la dictature a été très violente du point de vue des violations des droits de la personne. Par ailleurs, il est aussi connu que la torture existait bel et bien avant. Et que durant la dictature même, les tortures contre les criminels «communs» n'ont pas cessé d'avoir lieu. Le cas de l'«Escadron de la mort» à São Paulo (Esquadrão da morte) en est un exemple. Dans les années 1970, durant la dictature militaire, un groupe de policiers torturait et tuait des criminels ou tout simplement des accusés de crimes «communs», en prétextant un nettoyage de la ville de São Paulo. Au fil des investigations, on est venu à savoir que les morts, dont le nombre dépassait largement la centaine, étaient plutôt reliées à des réseaux de trafic de drogues et de prostitution, dont faisaient partie plusieurs agents de la police (Bicudo, 2006).

Néanmoins, c'est la violence à caractère politique qui a attiré l'attention sur le fait que la torture existait encore dans la société brésilienne. Peut-être ce changement est-il effectivement dû au fait que c'est uniquement quand les fils de la classe moyenne, les étudiants qui luttaient contre le régime, ont été capturés, torturés et tués par la «police politique» que le problème de la torture a été effectivement construit comme un problème. Le fait est qu'après la dictature, ce sujet a pris dans les préoccupations des gens un espace qu'il n'occupait pas avant.

Les points de cécité qui peuvent être créés sont justement les associations telles que: dictature - torture, ainsi que démocratie - garantie de la dignité bumaine. Même si personne n'aime consciemment le fait de penser à la démocratie en l'associant à la torture, le problème réside dans le fait que le régime politique en tant que tel ne garantit absolument 
pas la non-occurrence d'une telle pratique. On est face à une espèce de construction conceptuelle «aveugle» dans laquelle il y aurait une relation substantive entre les régimes politiques et la pratique (ou non) de la torture.

Max Weber (1919: 147) entend par «faits inconfortables» (« inconvenient facts») les faits qui peuvent se révéler désagréables à un observateur qui se rend compte que le point de vue qu'il considère comme juste commande d'agir contre ses propres valeurs. D'après Pires (2008), une des thèses qui semble être sous-jacente à la notion wébérienne de «faits inconfortables» est que ces faits, bien que phénoménologiquement assez visibles, ne seraient pas nécessairement faciles à voir pour ceux qui soutiennent une position pratique à l'égard de ces faits qui pourraient être inconfortables. Ces faits deviennent souvent des «taches aveugles» («blind spots») (von Foerster, 1973), c'est-à-dire des faits que l'on ne voit pas. Si j'adapte ces notions à la situation dont je traite dans cet article, je dirais que ceux qui adoptent une position contraire à la torture et luttent pour l'éradiquer peuvent éprouver de la difficulté à voir la coexistence entre démocratie et torture comme un «fait inconfortable».

\section{Conclusion}

Cette réflexion sur la difficulté à voir l'existence de la torture dans le régime démocratique m'amène à une autre réflexion, qui est peut-être à l'origine de la première: l'association, sur le plan conceptuel, entre démocratie et droits de la personne (Arat, 1999; Evans, 2001 ; Sjoberg, 2001 ; et au Brésil, Dimoulis, 2008; Ribeiro, 2008). La construction de cette association est observée tant dans les écrits universitaires que dans les discours des militants des droits de la personne, des politiciens et même des juristes. Cette approximation entre les deux concepts est tellement forte dans certains textes, qu'on trouve des formulations comme celle qui dit que la démocratie n'existe pas sans les droits de la personne et vice-versa (Dimoulis, 2008).

Sjoberg (2001) souligne qu'il faut garder à l'esprit que la démocratie et les droits de la personne expriment deux concepts différents, en dépit $\mathrm{du}$ fait qu'ils émergent sous la forme de phénomènes empiriques très marqués dans l'histoire récente de l'Occident. Mon hypothèse est qu'une partie importante de la difficulté à mieux formuler et décrire le problème de la torture dans les démocraties modernes occidentales réside 
dans le fait que sur le plan du discours, y compris du discours académique, la démocratie présuppose le respect et la protection des droits de la personne. Et vice-versa.

Or, la question qu'il faut poser est alors si cette «attitude cognitive» face aux concepts pose des problèmes - théoriques et/ou empiriques - et quelles sont ses conséquences. Dans un de ses articles, Arat (1999) affirme que les conceptualisations modernes de la démocratie sont toutes «connectées» aux droits de la personne; néanmoins, aucun régime démocratique n'a jamais atteint l'idéal de la protection égalitaire de la dignité humaine. De plus, empiriquement, la démocratie ne peut même pas éviter ou prévenir la violation des droits fondamentaux. Je vais accepter cette affirmation comme valide même en considérant des études comme celle de Davenport (1999) où il conclut que l'implantation et le développement de la démocratie peuvent expliquer la variation (diminution) des violations des droits de la personne (et non leur prévention).

Pour contribuer à cette discussion, j'aimerais développer brièvement deux aspects de la question. Le premier aspect renvoie aux manières de construire l'association entre démocratie et droits de la personne qu'on trouve dans la littérature. Le deuxième aspect concerne les difficultés - que je peux envisager pour l'instant - que cause cette association conceptuelle pour la pensée sur les droits de la personne.

Lorsqu'on observe la manière dont les textes, académiques ou non, abordent l'association entre démocratie et droits de la personne, il est possible de distinguer deux faces de l'association: la «face $1 »$ que je qualifierai comme plus descriptive et la «face $2 »$, comme plus normative. Dans la «face $1 »$, on trouve les formulations où les droits de la personne - ou au moins quelques-uns de ces droits - apparaissent comme une condition requise par la démocratie. J'ai qualifié cette face de «plus descriptive» dans le sens que, même dans l'acception le plus restrictive de la démocratie, ce que certains appellent «minimal democracy» (Diamond et Morlino, 2005), il faut effectivement présupposer quelques droits fondamentaux, notamment les droits politiques de vote, de liberté d'expression, d'association, etc. (Dahl, 1996).

Dans la «face 2 », on trouve des formulations où c'est la démocratie qui apparaît comme intégrant les droits de la personne (Cohen, 2008). La démocratie est vue ici, alors, comme une «évolution» des droits de la personne. Dans cette deuxième face ou deuxième manière d'associer les concepts, et où se trouve une bonne partie de la littérature de 
l'Amérique latine sur le sujet, les droits de la personne peuvent fonctionner de deux façons différentes: dans la première façon, les droits de la personne sont utilisés pour justifier et faire avancer la transition démocratique, c'est-à-dire qu'ils vont contribuer au passage du régime dictatorial au régime démocratique. Dans la deuxième façon, plus normative, les droits de la personne sont utilisés pour «améliorer» la qualité de la démocratie. Autrement dit, ils ne sont plus utilisés comme paramètres pour implanter la démocratie, mais pour l'évaluer comme une «bonne» ou une «mauvaise» démocratie. Diamond et Morlino (2005) vont dire que pour évaluer la qualité de la démocratie, il faut observer, entre autres, la dimension «liberté et égalité», ce qui inclut la garantie de certains droits, y compris des droits civils, politiques et sociaux.

Quels sont alors, pour la pensée et même pour la théorie des droits de la personne, les problèmes que cause cette deuxième façon de construire l'association des concepts? Lorsque ces derniers sont utilisés comme norme interne de la démocratie, comme paramètre du bon et du mauvais pour le fonctionnement du régime politique, les deux concepts (démocratie et droits de la personne) deviennent trop collés l'un à l'autre. L'évaluation de la démocratie devient «subjectivée» par les droits de la personne et la conséquence est qu'il devient de plus en plus difficile de les distinguer; autrement dit, on n'est plus capable de percevoir la «différence de la distinction», dans le sens de Bateson (1972).

Du côté de la démocratie (ou de la théorie démocratique), cette construction conceptuelle amène à une situation paradoxale où la démocratie ne peut jamais violer les droits de la personne sans devenir «moins démocratique». Les droits de la personne sont toujours associés à ce qui est bon, à ce qui est positif, de sorte que toutes les décisions contraires sont des décisions «non démocratiques». Donc, si la démocratie ne peut jamais prendre une décision «erronée» (contre les droits de la personne), on arrive à la conclusion que toutes les décisions démocratiques sont les «bonnes» décisions.

Du côté des droits de la personne, les problèmes que ce type d'association peut générer sont liés à un effet que je vais qualifier d'«opacification de la vision». La représentation des décisions démocratiques comme celles qui respectent les droits fondamentaux nous amène à croire que toutes les décisions démocratiques protègent et promeuvent effectivement les droits de la personne. Or, cette assomption peut être trompeuse et générer des taches aveugles pour les droits de la personne. 
Pensons à la peine de 30 ans de prison au Brésil, qui est la peine maximale déterminée par la législation (donc approuvée démocratiquement). Du point de vue exclusif de la dignité humaine, cette peine pose des problèmes qui ne sont tout simplement pas soulevés ou même pas vus par ceux qui mobilisent les droits de la personne pour revendiquer la protection d'autres droits.

Le fait que les droits de la personne sont toujours considérés comme ayant la «meilleure vision», y compris des décisions démocratiques, implique que ceux qui les mobilisent, doivent faire un effort spécial pour ne pas perdre leur capacité d'autocritique. Les décisions prises au nom des droits de la personne peuvent engendrer des situations paradoxales, et même contraires à certains aspects des droits de la personne. L'exemple du processus de criminalisation de la torture au Brésil l'illustre bien: les politiciens qui se disaient défenseurs des droits de la personne voulaient mettre des peines de prison tellement longues pour ce crime, qu'il a fallu le contrepoids du Parlement pour en empêcher la concrétisation (Possas, 2009). Autrement dit, les droits de la personne peuvent aussi être à la base de mauvaises décisions.

\section{Références}

Arat, Z. (1999). Human Rights and Democracy. Polity, 32 (1), 119-144.

Babissagana, E. (2006). L'interdit de la torture en procès? Bruxelles: Facultés Universitaires Saint-Louis.

Bateson, G. (1972). Steps to an Ecology of Mind. Northvale, New Jersey: Jason Aronson Inc.

Bicudo, H. (2006). Minhas mémorias. São Paulo: Martins Fontes.

Caldeira, T. P. R. (2000). City of walls: crime, segregation, and citizenship in São Paulo. Berkeley, California: University of California Press.

Cancelli, E. (1994). O mundo da violência: a polícia na Era Vargas. Brasília : Editora Universidade de Brasília.

Chevigny, P. (1999). Defining the role of police in Latin America. In J. Mendez, G. O'Donnell \& P. S. Pinheiro (Eds.), The (un) rule of law and the underprivileged in Latin America (49-70). Notre Dame: University of Notre Dame Press.

Cohen, J. (2008). Rethinking Human Rights, Democracy and Sovereignty in the Age of Globalization. Political theory, 36 (4), 578-606.

Costa, A. T. M. (2004). Entre a lei e a ordem: violência e reforma nas polícias do Rio de Janeiro e Nova York. Rio de Janeiro: Editora FGV.

Dahl, R. (1996). Democracy and Human Rights under Different Conditions of Development. In O. Savic (Ed.), The Politics of Human Rights (166-180). London, New York: Verso. 
Debuyst, Ch., Digneffe, F., \& Pires, A. P. (2008). Histoire des savoirs sur le crime et la peine (Vol 3). Bruxelles: De Boeck Université et Larcier.

Diamond, L., \& Morlino, L. (2005). Introduction. In L. Diamond \& L. Morlino (Eds.), Assessing the Quality of Democracy. Baltimore: John Hopkins University Press.

Dimoulis, D. (2008). Conflitos e complementaridade entre direitos humanos e democracia. Revista EMESC, 15 (21), 18-20.

Evans, T. (2001). If Democracy, then Human Rights? Third World Quarterly, 22 (4), 623-642.

Higgins, M., Haristos-Fatouros, M., \& Zimbardo, P. (2002). Violence Workers. Police Torturers and Murderers Reconstruct Brazilian Atrocities. Berkeley: University of California Press.

Holloway, T. (1993). Policing Rio de Janeiro: repression and resistance in a $19^{\text {th }}$ century city. Stanford, Calif. : Stanford University Press.

Human rights watch/Americas (1997). Police brutality in urban Brazil. New York, Washington.

Jacques, A. (1994). L'interdit ou la torture en procès. Paris: Les Éditions du Cerf.

Lehalle, S. (2008). La torture: une pratique en quête de légitimation. Texte présenté dans l'atelier du CIRCEM (Centre interdisciplinaire de recherche sur la citoyenneté et les minorités), Université d'Ottawa.

Mendez, J. E. (1999). Problems of lawless violence: introduction. In J. Mendez, G. O'Donnell, \& P. S. Pinheiro (Eds.), The (un) rule of law and the underprivileged in Latin America (19-24). Notre Dame: University of Notre Dame Press.

Pinheiro, P. S. (1999). The rule of law and the underprivileged in Latin America. In J. Mendez, G. O'Donnell, \& P. S. Pinheiro (Eds.), The (un) rule of law and the underprivileged in Latin America (1-18). Notre Dame: University of Notre Dame Press.

Pires, A. P. (1998). Aspects, traces et parcours de la rationalité pénale moderne. In C. Debuyst, F. Digneffe \& A. P. Pires (Eds.), Histoire des savoirs sur le crime et la peine (Vol. 2, 3-52). Ottawa: De Boeck Université.

Pires, A. P. (2008). Un «fait inconfortable»: les relations paradoxales entre droits de la personne et punition dans l'activité législative. Document de travail non publié, Chaire de recherche du Canada en traditions juridiques et rationalité pénale, Université d'Ottawa.

Pires, A. P., \& Garcia, M. (2007). Les relations entre les systèmes d'idées: droits de la personne et théories de la peine face à la peine de mort. In Y. Cartuyvels, H. Dumont, F. Ost, M. van de Kerchove \& S. Van Drooghenbroeck (Eds.), Les droits de l'bomme, bouclier ou épée du droit pénal? (291-336). Bruxelles: Bruylant et Facultés universitaires Saint-Louis (avec l'aide du Fonds de la recherche fondamentale collective).

Possas, M. T. (2009). Système d'idées et création de lois criminelles: le cas de la loi contre la torture au Brésil. Thèse de doctorat, Université d'Ottawa, Ottawa.

Ribeiro, R. J. (2008). Os direitos do homem podem ameaçar a democracia? In T. Rifiotis \& T. H. Rodrigues (Eds.), Educdção em Direitos Humanos: discursos críticos e temas contemporâneos. Florianópolis: Editora da UFSC. 
Sjoberg, G. (2001). A Sociology of Human Rights. Social Problems, 48 (1).

Soares, L. E., Batista, A., \& Pimentel, R. (2006). Elite da tropa. Rio de Janeiro: Objetiva.

Sutherland, E. H. (1949). White Collar Crime. New Haven: Yale University Press (édition de 1983).

Von Foerster, H. (1973). La construction d'une réalité. In P. Watzlawick (Ed.), L'invention de la réalité. Contributions au constructivisme. Paris: Seuil (édition de 1988).

Weber, M. (1919). Le savant et le politique. Paris: Plon (traduction de Julien Freund, édition de 1959).

ABSTRACT - Despite the fact that torture is on first inspection observed as an inacceptable practice in contemporary societies, when one looks further, two different discourses are identified: one that legitimates torture and another one that refuses it. This article will focus on the discourse that rejects torture and on the "construction of the sense of the problem" (Bachelard) of torture in Brazil and its explanations. These explanations are somewhat based on the belief that the persistence of torture in Brazil during the democratization period is due to its fragile democratic consolidation. This kind of reasoning carries some blind spots that prevent us from seeing important aspects, like the fact that torture is not the exclusive reality of authoritative regimes, but also, unfortunately, of democracies.

KEYWORDS - Torture, democratic consolidation, police violence, Brazil.

RESUMEN - A pesar de que en las sociedades occidentales contemporáneas la práctica de la tortura parece una práctica inaceptable, en cuanto se observa más de cerca el discurso a ese respecto se constata que existen de hecho dos discursos: uno que legitima la tortura y otro que la rechaza. El presente artículo analiza el discurso que rechaza esta práctica y la "construcción del sentido del problema" (Bachelard, 1938) de la tortura en Brasil y sus explicaciones. Estas últimas giran en torno a la creencia de que la persistencia de la práctica de la tortura en Brasil, luego del proceso de democratización en los años 1980, se debe a la débil consolidación de la democracia. Este tipo de razonamiento padece de un "punto ciego" que nos impide ver aspectos importantes, por ejemplo el hecho de que la práctica de la tortura no es en realidad exclusiva de los regímenes autoritarios, sino también, desafortunadamente, de las democracias.

PALABRAS CLAVE - Tortura, consolidación democrática, violencia policiaca, Brasil. 